\title{
Performance of the CMMB -MIMO System with TH Precoding Technology
}

\author{
Huiqin Wang \\ School of Information Engineering \\ Communication University of China \\ Beijing, China \\ hqwang@cuc.edu.cn
}

\begin{abstract}
The China Mobile Multimedia Broadcasting (CMMB) standard is the major standard in China for mobile multimedia broadcasting. It has been proved that the MIMO technology can increase the channel capacity remarkably comparing to traditional CMMB systems. However this technique plays a small role on the signal interference between multi-users. So the Tomlinson-Harashima precoding(THP) technology is introduced in the paper. The principle of the algorithm is elaborated for multiple-antenna and multi-user systems.The performance of CMMB-MIMO system with zeroforcing precoding is also analyzed on both channel capacity and BER in detail. As is shown in simulation results, the performances are better than those of conventional CMMB system after precoding algorithm is involved.
\end{abstract}

Keywords-CMMB-MIMO; THP; channel capacity

\section{INTRODUCTION}

The China Mobile Multimedia Broadcasting (CMMB) standard is the major standard in China for mobile multimedia broadcasting[1]. Up to now, the signal coverage rate of CMMB network has reached more than $98 \%$ in China. In order to adapt to the limited bandwidth of wireless channel, MIMO and Orthogonal Frequency Division Multiplexing (OFDM) modulation technology are introduced in CMMB system[2].MIMO utilizes multiple antennas to provide spatial diversity and multiplexing so as to obtain higher channel capacity, transmission reliability and spectral efficiency compared with traditional SISO system. While the original data can only be restored through complex detection techniques in MIMO system due to the inevitable interference existing among multiple data stream or users. Different from the MIMO detection performed at the receiver, precoding techniques use the channel state information to adjust the transmission strategy at transmitter to suppress the multiuser interference in MIMO channel ,improve the channel capacity and reduce the processing complexity of the receiver, which makes precoding techniques become the key factor in achieving multiplexing and diversity gain[3]. So THP technology is applied to the CMMB-MIMO system here. The performance about bit error rate and channel capacity of the new system are analyzed at the subsequent section and the last section shows simulation results of the CMMB -MIMO System with THP technology.

\section{SYSTEM MODEL}

MIMO channel has been described first in this section and THP scheme for multi-user CMMB-MIMO systems is discussed subsequently.

\section{A. CMMB-MIMO Channel model}

We consider a multi-user MIMO system with $N_{T}$ transmit antennas and $\mathrm{K}$ users,each user has $\mathrm{N}_{\mathrm{R}}$ receiver antennas . It is assumed that between each transmit and receive antenna a flat fading channel is present. The fading channel matrix $\boldsymbol{H}$ is independent, and identically distributed zero-mean complex Gaussian random variables with unit variance. Fig. 1 shows the channel model for the each user [4]. After channel coding, we use constellation mapping to map the bits into symbols with amplitude and phase information that are easy to modulate and transmit. Then we make the symbols joint together to form OFDM symbols in the frequency domain ${ }^{[3]}$. In allusion to these OFDM symbols, we use Alamouti coding structure and employ the same OFDM modulation for both the source and the encoded signals respectively. As far as $x_{\mathrm{i}}$ $\left(i=1,2, \ldots n_{\mathrm{T}}\right)$ is concerned,is the sending signal of the ith transmit antenna. And $r_{\mathrm{j}}\left(j=1,2, \ldots n_{\mathrm{R}}\right)$ is the receive signal of the $\mathrm{j}$-th receive antenna.Their relation can be presented by

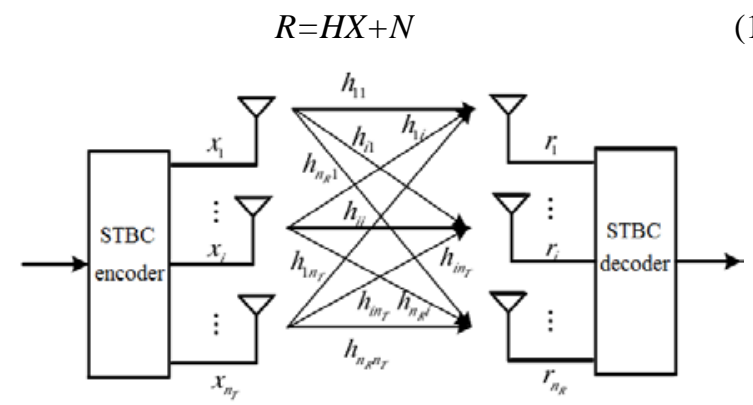

Fig. 1. Systm model for CMMB-MIMO channel

Where $N=\left[N_{1}, N_{2}, \ldots, N_{n R}\right]$ is the noise vector at receivers with independent elements. It can be found that multi-user interference to the receiver signal would be inevitably introduced. Therefore, how to eliminate the interference become a severe matter. 


\section{B. CMMB-MIMO THP}

The block diagram of THP for multi-user CMMB-MIMO system is shown in Fig.2.

Column vector $U=\left[d_{1}, d_{2}, \ldots, d_{\mathrm{K}}\right]$ consists of all users'

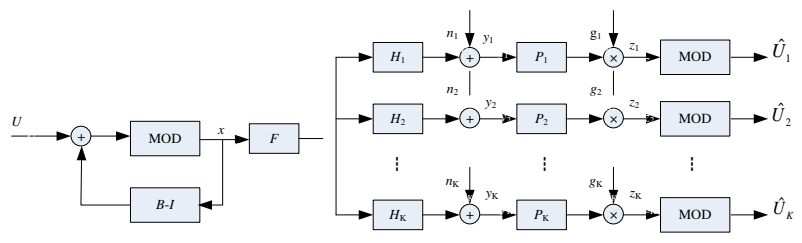

Fig. 2. THP for multi-user CMMB-MIMO system signals, where $d_{\mathrm{k}} \in \mathrm{C}^{n R \times 1}$, which are obtained from the 16QAM data sets. $H_{\mathrm{k}} \in \mathrm{C}^{n R \times n T}$ is the channel model of kth user's. $P_{\mathrm{k}}$ $\in \mathrm{C}^{n R \times n R}$ is the predisposing matrix of kth user's. $n_{\mathrm{K}} \in \mathrm{C}^{\mathrm{nR} \times 1}$ is the Gaussian white noise vector at receivers of $\mathrm{k}$-th user[4]. Transmitter-based feedback matrix $B \in C^{\mathrm{nT \times nT}}$ is used to eliminated the interferences between users,receive signals $Z_{\mathrm{k}}$ $\in \mathrm{C}^{\mathrm{nR} \times 1}$. The transmitted symbols $d_{\mathrm{K}}$ are successively calculated via the modulo arithmetic feedback filter. As to16QAM modulation, the result of modulo arithmetic $\in[-$ $4,4]$,such that a transmit signal has smaller power. At the receiver, which applies the same modulo operation as that at the transmitter, is used.

It is assumed that for $H$ to be known perfectly at receiver and transmitter , $H$ is the channel matrix, defined as (2)

$$
H=\left[H_{1}, H_{2}, \ldots, H_{K}\right]^{\mathrm{T}}
$$

It should be factorized through QR decompisition $H^{H}=Q R$, where $R$ is an upper triangular matrix ,and $r_{\mathrm{ii}}\left(i=1,2, \ldots n_{\mathrm{R}}\right)$ is the diagonal elements of $R, Q$ is a unitary matrix. The relations between $F, B$ and $R, Q$ are presented as follows[5]:

$$
\begin{gathered}
G=\operatorname{diag}\left(1 / r_{11}, 1 / r_{22}, \ldots\right) \\
F=Q^{\mathrm{H}} \\
B=G R^{\mathrm{H}} \\
x=B^{-1} U
\end{gathered}
$$

At receiver side the received signal for transmission is expressed as

$$
Z=G P^{\mathrm{H}} H F x+G P^{\mathrm{H}} n
$$

Where $P$ is a block diagonal matrix, $P=\operatorname{diag}\left(p_{1}, p_{2}, \ldots, p\right.$ ${ }_{\mathrm{K}}$ ), where $p_{\mathrm{K}}$ is the eigenvector of $\mathrm{k}$-th maximum eigenvalues of matrix $H_{k} H_{k}^{H}$. Here $P$ is taken as an identical matrix.

According to (4) to (7),the received sigal fo each user can be expressed by

$$
Z=G P^{H} H\left(F B^{-1}(U+\lambda)+n\right)=U+\lambda+\tilde{n}
$$

Where $\tilde{n}=G n$ denotes the filtered channel noise.

At last, the received data can be expressed as

$$
\hat{U}=\bmod _{2 \sqrt{M}}(Z)=\bmod _{2 \sqrt{M}}(U+\lambda+\tilde{n})=U+\tilde{n}
$$

Therefore $\mathrm{TH}$ precoding can effectively eliminate multiuser interference and improve the system throughput.

\section{Channel Capacity of THP CMMB system}

The channel capacity of CMMB system with TH precoding can be calculated as follows. From the block diagram shown in Fig. 2, at the transmitter, the estimated channel can be expressed as:

$$
\hat{H}=\left[\hat{H}_{1}, \hat{H}_{2}, \ldots, \hat{H}_{K}\right]^{T}
$$

QR decompisiton is preformed for $\hat{H}$ and (10) is obtained

$$
\hat{H}^{H}=Q R
$$

At receiver side signal is given by

$$
\begin{aligned}
y & =H F X+n \\
& =(\hat{H}+\Delta) F X+n \\
& =R^{H} F X+\Delta F X+n
\end{aligned}
$$

where $\Delta=H-\hat{H}$ is the difference between the estimated channel and the original channel[6]. Then we define $\Delta_{k}=H_{k}-\hat{H}_{k}$. Received signal of k-th user can also be expressed as

$$
y_{k}=r_{k k} x+\sum_{k<j} r_{k j} x_{j}+\Delta_{k}^{T} F X+n_{k}
$$

where $\sum_{k<j} r_{k j} x_{j}$ is taken into account as the known interference according to the procedure of THP. It is not regarded as interference any more. Therefore, the SNR for kth user can be calculated as

$$
S N R_{k}=\frac{r_{k k}^{2} P_{0} / K}{1+\left\|\Delta_{k}\right\|^{2} P_{0} / K}
$$

where $P_{0}$ represents the transmit signal power. The channel capacity of CMMB system is given by

$$
C_{\text {THP }}=\sum_{k=1}^{K} E\left\{\log _{2}\left(\frac{r_{k k}^{2} P_{0} / K}{1+\left\|\Delta_{k}\right\|^{2} P_{0} / K}\right)\right\}
$$

\section{SIMULATION RESULT}

The overall system implementation was used for simulation. It used random bit sequence generation for bitstream mapping. The selected values for CMMB modulation in all the simulations are the following ones.

1) Transmission mode: $8 \mathrm{M}$

2) Constellation: 16QAM.

Rayleigh Fading channel is commonly used as a reference channel model for performance analysis of MIMO systems due to its simplicity. Given that the MIMO system under study is $4 \times 4$, and there are 4 users. 
Then, the result comparison of the channel capacity of THP and conventional CMMB-MIMO system is presented as follows.

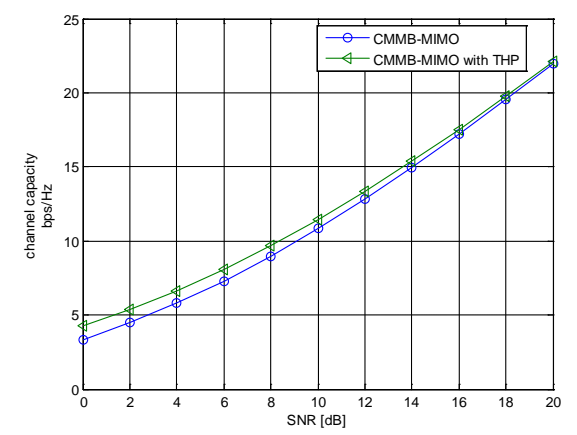

Fig .3. Comparison of channel capacity between two schemes

As shown in Fig. 3, the channel capacity of THP is better than that of CMMB-MIMO system when SNR is low. But when SNR is high, the difference between two schemes is gradually small with SNR increasing. There are two reasons about this case, firstly obtaining channel state information plays a small role on channel capacity. Secondly THP algorithm results in some capacity damage due to the waveform of receivers.

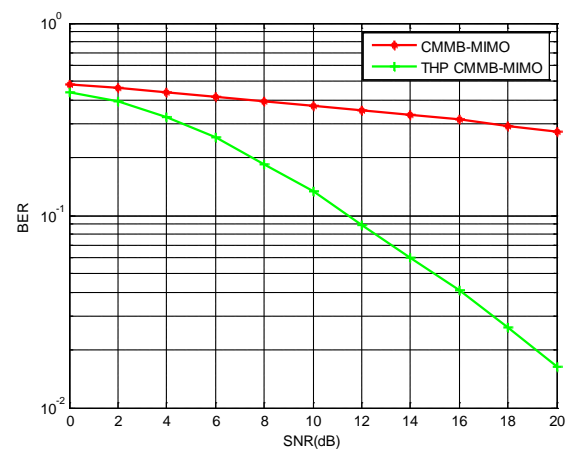

Fig.4. Comparison of BER between two schemes

As described in Section II, the performance of THP is determined by the minimum absolute diagonal elements of matrix $R$. The comparison of BER performance is shown in Fig. 4. The performance of BER is improving greatly using THP technology than that without it, especially on high SNR. And the gap between two schemes widens with SNR increasing, which mainly because TH precoding can effectively eliminate multi-user interferences .

\section{CONCLUSION}

In this paper, THP technology is introduced in CMMB MIMO system. At the following sections, the principle of force-zero THP is demonstrated for multiple-antenna and multi-user system, and the performance on channel capacity and BER of the new scheme are analyzed. Finally through the computer simulation, we can draw a conclusion that compared to the conventional CMMB system, the new scheme has obvious advantage on both aspects.

\section{REFERENCES}

[1] GY/T 220.1-2006, Mobile Multimedia Broadcasting Part 1: Frame Structure, Channel Coding and Modulation for Broadcasting Channel, THE STATE ADMINISTRATION OF RADIO FILM ANDTELEVISION, 2006.11

[2] Feng. Hu, Libiao.Jin, and Jianzeng.Li, "Performance of the CMMB System with MIMO Transmission Scheme,” 2012 Second International Conference on Instrumentation \& Measurement, Computer, Communication and Control, pp.1041-1044, 2012.

[3] Jun. Xu, Raju.Hormis, and Xiaodong. Wang,”MIMO video Broadcast via Transmit Precoding and SNR-Scalable Video Coding, " IEEE Journal on Selected Areas in Commucinations. vol. 28, No. 3, April 2010

[4] S. Zazo and H. Huang, “ Suboptimum space multiplexing structure combining dirty paper coding and receive beamforming, "ICASSP, Toulouse, France, vol. 4, pp.89-92, 2006.

[5] H. Khaleghi Bizaki and Abolfazl Falahati. "Tomlinson-Harashima Precoding with Imperfect Channel Side Information,” IEEE ICAC. Vol. 2, pp.987-991, 2007.

[6] Guoxian.Lu,Muqing.Wu,Si.Zhou,andFeng.Zheng, "TomlinsonHarashima Precoding Optimization for Multi-User MIMO Systems in Indoor Scenarios, ”2012 IEEE $14^{\text {th }}$ International Conference on Communication Technology , pp. 1062-1066,2012. 